\title{
ESTUDIO EXPLORATORIO DE FACTORES MOTIVACIONALES INTERNOS Y EXTERNOS, Y SU RELACIÓN CON EL DESEMPEÑO DOCENTE EN LA PONTIFICIA UNIVERSIDAD CATÓLICA DEL ECUADOR (PUCE)
}

\author{
INTERNAL AND EXTERNAL MOTIVATIONAL FACTORS AND THEIR INFLUENCE \\ ON FACULTY PERFORMANCE AT THE PONTIFICA UNIVERSIDAD CATÓLICA \\ DEL ECUADOR
}

Christian Cabezas ${ }^{1}$ Maria Cristina Ramos ${ }^{2}$

Pontificia Universidad Católica del Ecuador (PUCE)

(RECIBIDO 10/4/2012; ACEPTADO EL 02/7/2012)

\begin{abstract}
RESUMEN
Este estudio exploratorio analizó los diez factores relacionados con la motivación hacia el trabajo y la satisfacción laboral propuestos por Herzberg, Mausner y Snyderman (1959) y su influencia en el desempeño docente en una muestra representativa de profesores de la Pontificia Universidad Católica del Ecuador. Entre los factores se incluyeron características propias del trabajo (factores internos) así como características propias del contexto en el que este se desarrolla (factores externos). A su vez se examinó la diferencia que los docentes tuvieron en los factores motivacionales de acuerdo a sus características tales como género, categoría docente y dedicación docente. Al finalizar el estudio, los análisis estadísticos identificaron al factor externo "supervisión en el trabajo" como predictor del desempeño docente. Además se hallaron diferencias con significación estadística en factores específicos de acuerdo al grupo de dedicación y a la categoría de los profesores. Adicionalmente, se encontró una diferencia con significación estadística en el factor "trabajo en sí" dependiendo del género de los profesores. Las profesoras mostraron un nivel de satisfacción más alto en este factor que los profesores. Estos hallazgos llaman a profundizar en siguientes investigaciones sobre estas variables específicas.
\end{abstract}

Palabras clave: motivación, factores motivacionales, desempeño docente, satisfacción laboral

1 Christian Cabezas Guerra (Autor del Proyecto): Obtuvo su título de Psicólogo en la PUCE. Posteriormente se graduó de Máster en Psicología (MSc in Psychology) en la Universidad de Idaho (Estados Unidos). También obtuvo el título de Maestría en Administración y Gestión de Negocios en la Universidad de Alcalá de Henares (Madrid- España). Actualmente docente en la Pontificia Universidad Católica del Ecuador.

2 MaríaCristina Ramos Flor (Colaboradora): Obtuvo su título de Psicóloga Organizacional en la PUCE. Ha colaborado en varios proyectos de investigación sobre temas de educación y psicología durante los años 2009, 2010 y 2011. 


\begin{abstract}
This exploratory study analyzed ten factors related to motivation towards job satisfaction, as proposed by Herzberg, Mausner, and Snyderman (1959), and their influence on faculty performance in a representative sample of faculty members of the Pontifical Catholic University of Ecuador between 2010 and 2011.

These factors included work characteristics (internal factors) as well as context characteristics where work is performed (external factors). The effect that some faculty members' characteristics, such as faculty time category (i.e. full-time, half-time, part-time), position category, and gender had on job satisfaction and on the motivational factors was also analyzed. Statistical analysis showed that the external factor "job supervision" was a significant predictor of faculty performance. Statistically significant differences were found in some specific motivational factors, depending on the faculty time and position categories. Another statistically significant difference was discovered in the factor "work itself" depending on the faculty gender. Female faculty members showed a higher level of satisfaction in the work itself category than male faculty members.
\end{abstract}

Keywords: Motivation, motivational factors, faculty performance, job satisfaction

\title{
INTRODUCCIÓN
}

La satisfacción laboral, es decir, las emociones positivas o placenteras que resultan de la experiencia de una persona producto de su trabajo (Locke, 1976), es uno de los constructos más ampliamente estudiados por la psicología organizacional. Esto se debe al impacto que esta variable puede tener en el bienestar de individuos, grupos y organizaciones. Ciertas investigaciones identifican a la satisfacción laboral como un factor capaz de influir en el desempeño (Judge, Thoresen, Bono, y Patton, 2001), la retención de personal (Johnsrud y Heck, 1994; Rausch et al., 1989; Hagedorn 1996; Rosser 2004; Smart 1990; Ostrof, 1992), el absentismo (Hackett y Guion, 1985) y la ciudadanía organizacional (Organ y Ryan, 1995). Por esta razón, organizaciones de todo tipo requieren identificar los factores subyacentes a la satisfacción laboral para así dirigir sus esfuerzos de manera efectiva hacia el aumento de dicha satisfacción.

El estudio de la satisfacción laboral ha tenido lugar desde 1911, año en que Frederick Taylor planteó que la satisfacción laboral depende únicamente del salario percibido (Kanigel, 1997). De acuerdo a esta perspectiva, mientras más salario percibe una persona, más motivación encontrará para realizar su trabajo. Años más tarde, Brayfield y Crockett (1955) concluyeron que la satisfacción laboral depende en gran medida de las condiciones de trabajo y las necesidades y expectativas del trabajador. Como respuesta a estos planteamientos, en 1959 Frederick Herzberg y sus colaboradores desarrollaron una teoría en la que se explican diez factores o variables susceptibles de medición que están relacionadas con la satisfacción y la insatisfacción laboral. Estos factores se encuentran agrupados en dos conjuntos. 
El primer grupo de factores incluye variables que facilitan la satisfacción laboral, por lo que son denominados factores motivadores. En este grupo se incluyen los factores: logro, reconocimiento, el trabajo en sí, responsabilidad y crecimiento. Todas estas variables se relacionan con características inherentes al cargo y las tareas que se deben realizar, por lo que se considera a estos factores internos. Por el otro lado, están las variables relacionadas con la insatisfacción laboral que se denominan factores higiénicos. A este grupo pertenecen las políticas y administración de la institución, supervisión, salario, relaciones interpersonales y condiciones de trabajo. Los factores higiénicos se relacionan con características del contexto en el que se realiza el trabajo, por lo que constituyen factores externos. Según Herzberg (1959), si los factores externos no son los adecuados se produce insatisfacción laboral. Cuando los factores externos se encuentran en condiciones óptimas (p.ej. salario competitivo) evitan la insatisfacción del personal, mas no producen satisfacción laboral. Por el contrario, los factores internos consiguen aumentar el grado de satisfacción laboral. Para este autor, el contrario de la insatisfacción laboral no es la satisfacción sino más bien la no insatisfacción. De la misma manera, el opuesto de la satisfacción laboral es la no satisfacción laboral (Herzberg, Mausner y Snyderman, 1959).

Se han realizado investigaciones que han examinado la satisfacción laboral en instituciones educativas a través de la teoría de los dos factores de Herzberg (Diener, 1985; Hill, 1986; Rosser, 2004 y 2005; Mamiseishvili, 2011). En general, estos estudios sugieren que los docentes universitarios se encuentran altamente motivados por los factores intrínsecos, especialmente por el factor "trabajo en sí". Otras investigaciones sugieren que la satisfacción laboral incrementa con la edad y que puede disminuir según circunstancias familiares de los docentes tales como separaciones entre miembros de la familia y divorcio (Hagedorn, 2000). Otros estudios indican que al examinar las diferencias en el grado de satisfacción laboral según género, las mujeres reportaron estar menos satisfechas con su trabajo como docentes universitarias en comparación a sus colegas hombres (Aguirre 2000; Hagedorn 2000; Astin et al. 1997; Johnsrud y Sadao 1998; Rosser 2005; Smart 1990; Perna 2001; Tack y Patitu 1992; Trower y Chait 2002; Olsen et al. 1995; Turner y Myers 2000; Turner 2002).

Se debe notar que la gran mayoría de estos estudios se han realizado en países como Estados Unidos, generando inquietudes sobre posibles diferencias entre los resultados obtenidos en dichas investigaciones y los que se podrían obtener al examinar en instituciones educativas latinoamericanas. Por esta razón se halló pertinente estudiar la satisfacción laboral en instituciones de educación superior dentro del contexto latinoamericano. El estudio realizado buscó hallar los efectos de los factores externos e internos de motivación en la satisfacción laboral y el desempeño de los docentes de la Pontificia Universidad Católica del Ecuador (PUCE). Además, se analizó el efecto que las características docentes como género, categoría, dedicación, tienen sobre el grado de satisfacción con estos mismos factores y el desempeño docente. 


\section{OBJETIVO DE LA INVESTIGACIÓN}

Esta investigación tuvo como objetivo principal explorar el efecto que los factores motivacionales internos y externos tienen sobre el desempeño de los docentes medido a través de la evaluación docente en una muestra significativa de profesores de la PUCE. Adicionalmente se exploró el efecto que algunas variables categóricas (género, categoría docente y dedicación docente) pudiesen tener sobre factores motivacionales específicos.

Los objetivos de este estudio exploratorio fueron los siguientes:

- Explorar y analizar diferencias en el nivel de satisfacción de los docentes en cuanto a factores motivacionales en general (estadísticas descriptivas).

- Explorar y analizar diferencia en el nivel de satisfacción de los docentes en cuanto a factores motivacionales en relación a la dedicación, categoría, y género de los profesores.

- Analizar el efecto de factores motivacionales internos y externos de los docentes sobre su desempeño.

- Analizar el efecto de algunas variables especificas de los docentes sobre satisfacción de laboral de los docentes.

\section{MÉTODO}

\section{Variables y medición}

Por la naturaleza de esta investigación exploratoria se colocaron las variables dependientes e independientes de una manera flexible para responder a las preguntas de la investigación.

En un segmento inicial de esta investigación, la variable dependiente fue el "desempeño docente" medido a través de los resultados de la evaluación docente realizada por los estudiantes perteneciente al Segundo Semestre 2009-2010 y primer semestre 2010-2011. Las variables independientes se derivaron de la medición de 10 factores relevantes relacionados con la satisfacción e insatisfacción laboral. Estos factores se midieron empleando una versión del "Wood's Faculty Satisfaction/Dissatisfaction Scale" traducida y adaptada por los autores de este estudio. Este cuestionario consta de 59 ítems puntuables en una escala del 1 (muy insatisfecho) al 5 (muy satisfecho) que permiten obtener una medida de cada uno de los 10 factores internos y externos expuestos en la teoría de Herzberg (1959). Además, existió un ítem que mide la satisfacción general con respecto al trabajo que el docente desempeña.

En un segundo segmento de esta investigación se colocaron para el análisis las características propias de los profesores (género, dedicación, y categoría) como variables que hipotéticamente podían tener un efecto de diferenciación en los factores motivacionales. Es decir, se midió la diferencia de que existía en los resultados de los factores motivacionales dependiendo de estas variables categóricas. 


\section{Muestra y segmentación}

En esta investigación se utilizó una muestra conformada por un segmento representativo de docentes de la PUCE pertenecientes a 14 unidades académicas. Se tomó aproximadamente un $8 \%$ del total de profesores de cada unidad académica para la investigación. Un total de 78 profesores participaron en este estudio. A continuación se presentan los datos numéricos de la muestra obtenida.

TABLA 1: Proporción de muestra de docentes por unidad académica

MUESTRA: TABLA DE FRECUENCIAS POR FACULTADES

\begin{tabular}{l|c|c}
\multicolumn{1}{c|}{ FACULTAD } & MUESTRA & $\begin{array}{c}\text { TOTAL DO- } \\
\text { CENTES }\end{array}$ \\
\hline Arquitectura, Diseño y Artes & 8 & 104 \\
\hline Bioanálisis & 2 & 22 \\
\hline Ciencias Administrativas y Contables & 12 & 146 \\
\hline Ciencias de la Educación & 1 & 14 \\
\hline Ciencias Exactas y Naturales & 4 & 52 \\
\hline Ciencias Humanas & 6 & 88 \\
\hline Ciencias Filosófico-Teológicas & 2 & 26 \\
\hline Comunicación, Lingüística y Literatura & 18 & 163 \\
\hline Economía & 4 & 47 \\
\hline Enfermería & 4 & 48 \\
\hline Ingeniería & 6 & 80 \\
\hline Jurisprudencia & 7 & 38 \\
\hline Psicología & 3 & \\
\hline Trabajo Social & 78 & \\
\hline & & 7 \\
\hline
\end{tabular}

\section{RESULTADOS}

\section{Diferencias de satisfacción en cuanto a factores motivacionales en general}

En la fase inicial de este análisis se obtuvieron estadísticas descriptivas en lo que respecta a los promedios que todos los docentes de la muestra tuvieron en los diez factores motivacionales (internos y externos) 
Los resultados de los factores de motivación interna se presentan en la figura número $1 \mathrm{y}$ de los factores externos en la figura número 2 .

Figura 1: Resultados de los factores motivacionales internos de los profesores de la PUCE

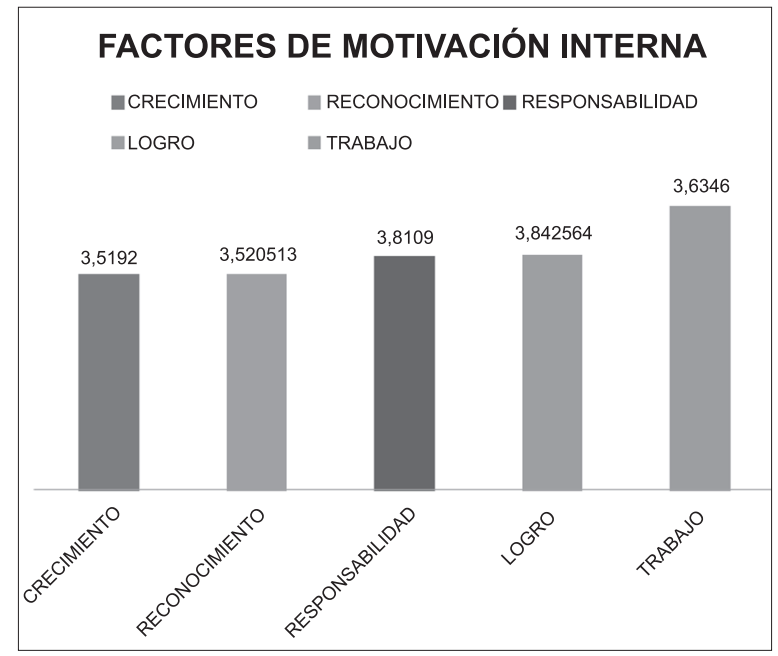

Figura 2: Resultados de los factores motivacionales externos de los profesores de la PUCE

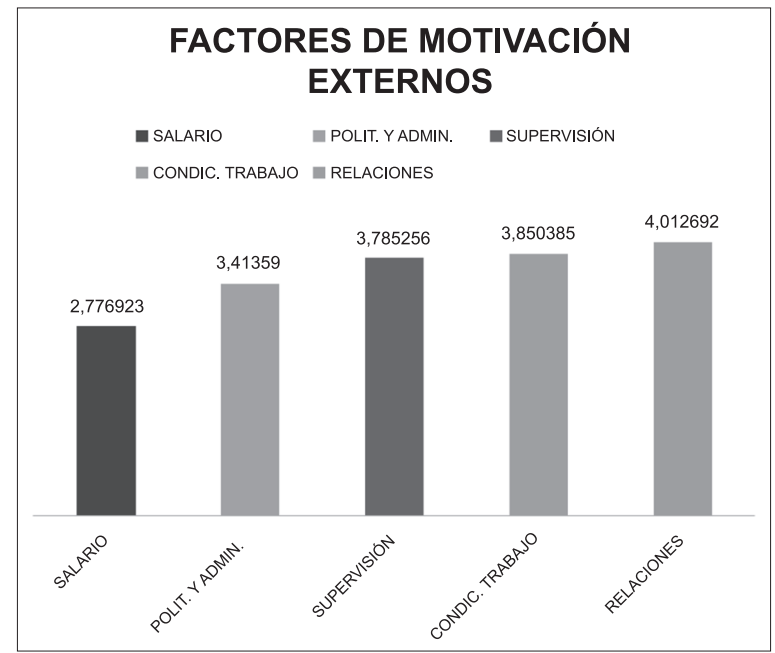

\section{Diferencia de factores en relación a la categoría de los profesores}

Los resultados de las estadísticas descriptivas mostraron que los profesores principales tuvieron niveles superiores de satisfacción en la gran mayoría de factores motivacionales tanto internos como externos. En lo que respecta a los factores generales y la satisfacción en general, en la figura 3 se puede apreciar que los profesores principales tuvieron niveles más altos de satisfacción general, así como en los factores internos y externos en general. 
Figura 3: Resultados de los factores de motivación generales y satisfacción por categoría

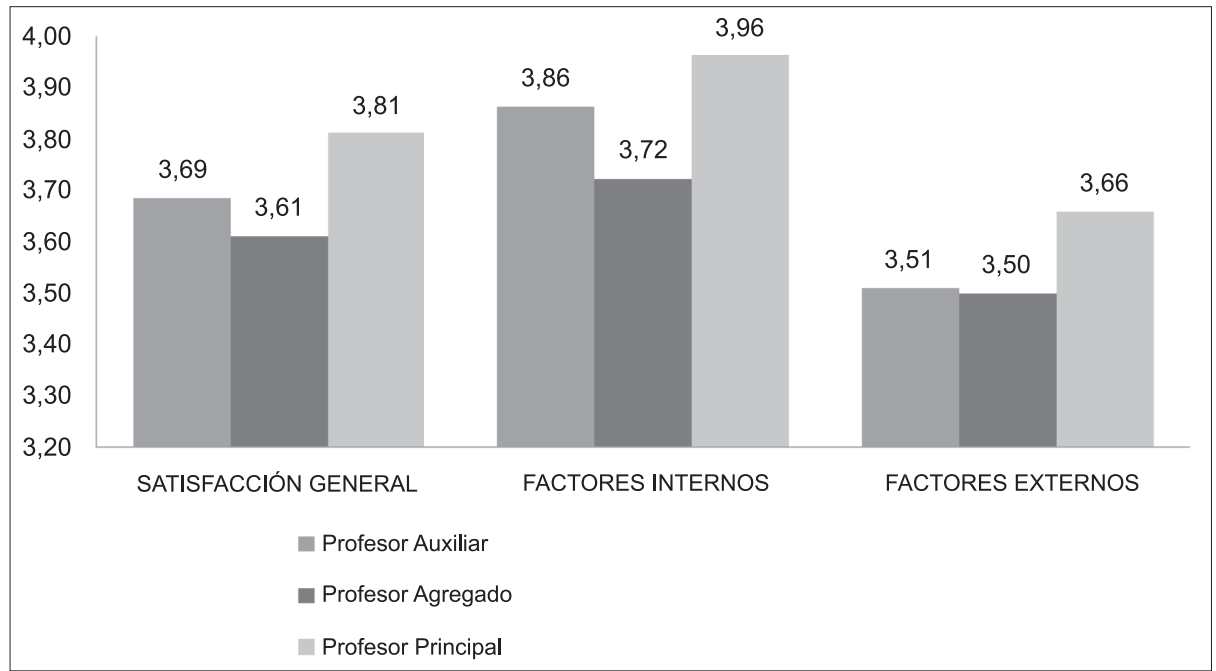

\section{Diferencia de factores en relación a la dedicación de los profesores}

En cuanto al tipo de dedicación de los profesores se pudo apreciar en las estadísticas descriptivas que los profesores a tiempo completo tuvieron puntuaciones más altas que los profesores de los demás tipos de dedicación en todos los factores motivacionales tanto internos como externos. En lo que respecta a los factores generales y la satisfacción en general, en el gráfico 4 se puede apreciar que los profesores a tiempo completo tuvieron puntajes más altos en la satisfacción general, así como en los factores internos y externos en general.

Figura 4: Resultados de los factores de motivación generales y satisfacción por dedicación

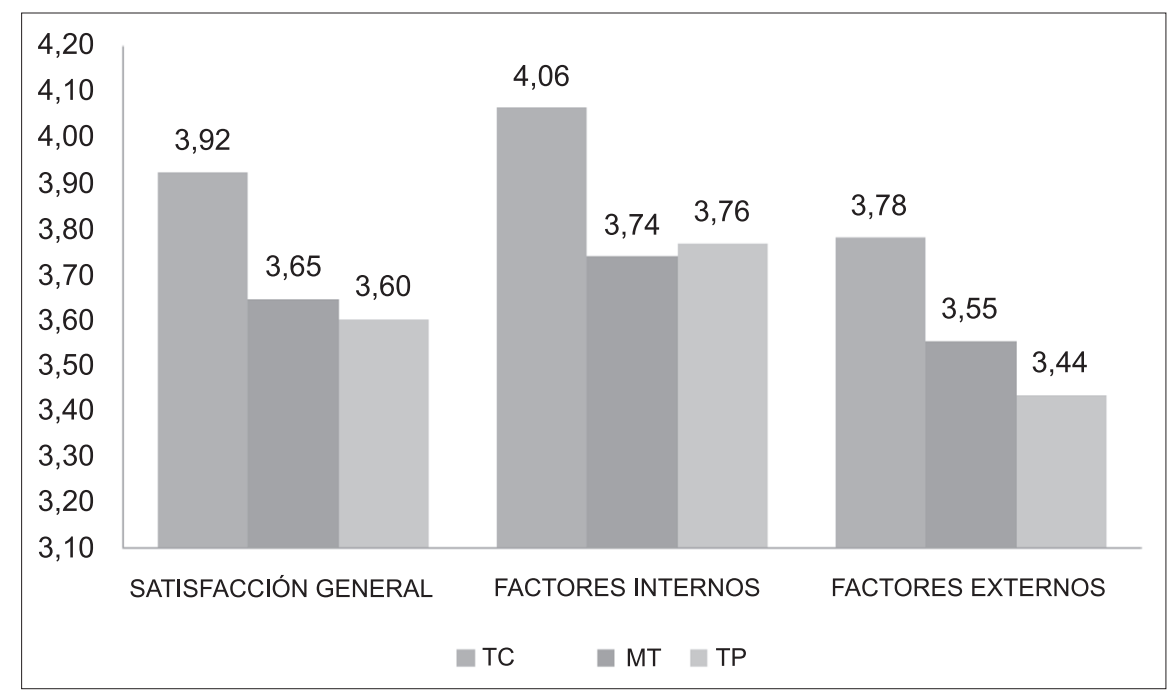




\section{Diferencia de factores en relación al género de los profesores}

De los resultados de las estadísticas descriptivas se pudo apreciar que las docentes mujeres tuvieron niveles más altos en todos los factores motivacionales internos y externos con excepción del factor "relaciones interpersonales".

Consistente con estos hallazgos, en la figura 5 se presentan los resultados mostrando que las docentes mujeres tuvieron un nivel mayor de satisfacción en los factores generales y en la satisfacción en general que los docentes varones.

Figura 5: Resultados de los factores de motivación generales y satisfacción por género

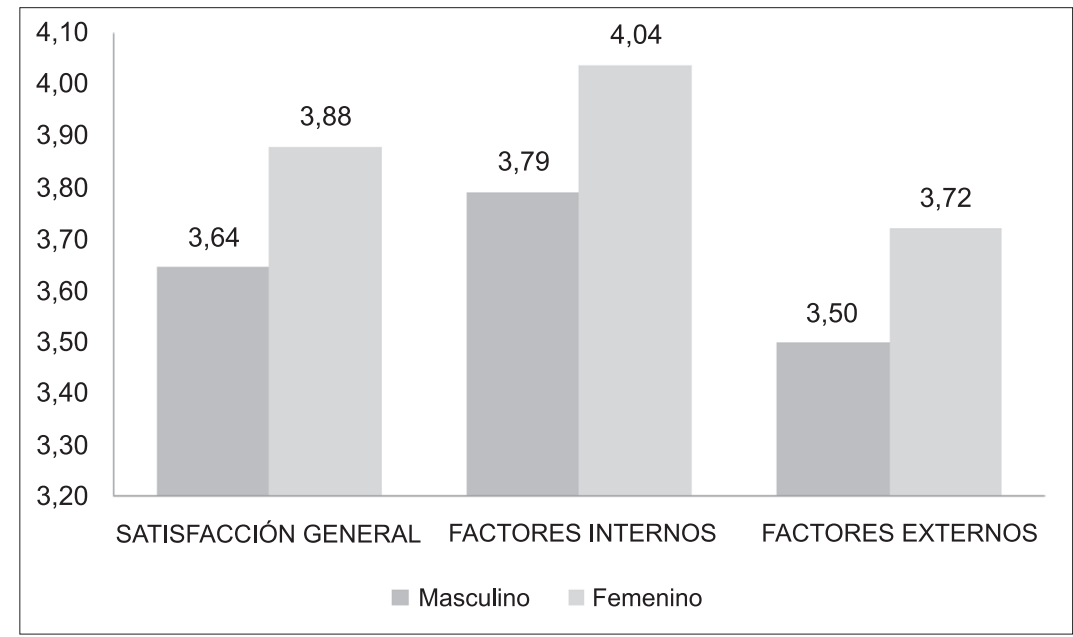

\section{Influencia de cada factor motivacional sobre el desempeño docente}

Realizando un análisis independiente de cada uno de los diez factores como predictores de desempeño docente, mediante regresión lineal simple, se encontró que la gran mayoría de factores independientemente analizados mostraron una escasa influencia sobre el desempeño docente con coeficientes de determinación menores a 0.05. Estos resultados tampoco contaron con significación estadística.

El único factor que en este análisis específico tuvo un rol llamativo como predictor del desempeño docente fue el factor externo supervisión. Este factor resultó ser predictor con significación estadística del desempeño docente con un coeficiente de determinación corregido de 0.065, y una significación de p. $<0.05$.

\section{Influencia de todos los factores motivacionales en conjunto sobre el desem- peño docente}

Para determinar la influencia que podrían tener los diferentes factores motivacionales internos y externos en conjunto sobre el desempeño docente se realizó un análisis de regresión múltiple en el que se ubicó a todos los factores motivacionales como predictores del desempeño docente. Como resultado general se encontró que los diez factores tuvieron un coeficiente de determinación corregido de 0,29 como predictores del desempeño docente. 
Posteriormente se desarrolló un análisis de regresión por pasos (stepwise) para determinar cuáles eran los factores que más afectaban el desempeño docente. Los factores supervisión, logro, crecimiento, relaciones y condiciones de trabajo en conjunto, y en ese orden, según este análisis, pudieron predecir el desempeño docente en conjunto en un $29 \%$ (coeficiente de determinación corregido).

Es decir que los cinco factores encontrados en este análisis por pasos pueden llegar a tener el mismo nivel de predicción que los 10 factores en conjunto. Por esa razón, de acuerdo a este estudio, esos cinco factores son los más importantes en lo que respecta a influencia en el desempeño docente.

\section{Efecto de remuneración sobre la satisfacción docente en general}

Como este estudio era exploratorio en naturaleza, se intentó indagar sobre el efecto que podría tener la remuneración de los profesores en la satisfacción laboral general medida a través de un indicador general. Para este fin, con los datos individuales de los docentes en lo que respecta a su dedicación, carga horaria y categoría, se pudo establecer su salario real. Posteriormente, se realizó un análisis de regresión para indagar sobre la influencia del salario real en la satisfacción general de los profesores.

Del análisis de regresión simple se pudo desprender que el salario real tiene una moderada influencia sobre la satisfacción laboral de los profesores con un coeficiente de determinación corregido de 0,037. Este análisis tuvo significación de p. $=0,049$.

\section{Diferencia en factores motivacionales de acuerdo a variables categóricas (dedicación, categoría y género)}

Puesto que se encontraron diferencias perceptibles en las estadísticas descriptivas en los factores motivacionales dependiendo de la dedicación horaria, la categoría docente y el género, se procedió a realizar algunos análisis para identificar si estas diferencias contaban con significación estadística.

\section{Diferencia en factores motivacionales de acuerdo a dedicación}

Dado que existe en la PUCE dedicaciones específicas docentes que son otorgadas a través del tiempo de servicio, también se investigó el efecto que el pertenecer a estas dedicaciones tendría sobre factores tales como la motivación interna y externa, y la satisfacción general.

Para este efecto se dividió inicialmente a los docentes en dos grupos: profesores a tiempo parcial, quienes tienen una dedicación menor y variable, y profesores a medio tiempo y tiempo completo. Estos dos grupos tienen conceptualmente una dedicación diferente de tiempo dado que los docentes al alcanzar la dedicación de medio tiempo y tiempo completo tienen una dedicación mayor y constante contractualmente.

Realizando la prueba t de comparación de medias se encontró que existía una diferencia con significación estadística entre los dos grupos divididos de dedicación 
en lo que respecta a su motivación general $(\mathrm{p} .=0,48)$, y su satisfacción con los factores externos (p. $=0,43)$.

Posteriormente se realizaron análisis de varianza para examinar si existía una diferencia con significancia estadística en cada factor motivacional dependiendo del tipo de dedicación. Se encontró esta diferencia en los factores crecimiento $(\mathrm{p} .=0,03)$, salario $(\mathrm{p} .=0,03)$ y condiciones de trabajo $(\mathrm{p} .=0,02)$. Para identificar entre que grupos existía la diferencia se realizó el test Tukey, el cual arrojó como resultados que la misma existía entre los grupos de tiempo completo y tiempo parcial.

\section{Diferencia en factores motivacionales de acuerdo a categoría docente}

No se encontraron diferencias con significación estadística en lo que respecta a la satisfacción en general ni en los factores generales de motivación interna y externa. No obstante, en el análisis individual de factores internos y externos se encontró una diferencia con significación estadística en el factor "condiciones de trabajo" dependiendo de la categoría de los profesores $(\mathrm{p} .=0,025)$. El análisis posterior utilizando el test Tukey mostró que la diferencia existía entre los profesores principales y los auxiliares.

\section{Diferencia en factores motivacionales de acuerdo a género}

Utilizando la prueba t de comparación de medias no se encontró una diferencia con significación estadística entre géneros en lo que respecta al indicador de satisfacción general, ni tampoco en los factores generales internos y externos.

Llamó la atención haber encontrado una diferencia con significación estadística entre géneros en el factor trabajo en sí $(\mathrm{p} .=0,05)$, teniendo las docentes mujeres un puntaje superior (más satisfacción) en este factor que los docentes varones.

\section{DISCUSIÓN}

De los resultados de esta investigación se desprende que en la institución donde se realizó, existe un grado superior de satisfacción de los profesores en cuanto al factor interno "trabajo en sí" comparado con los otros factores internos. Este resultado es consistente con lo encontrado en diferentes instituciones educativas y se ajusta a lo usualmente considerado en la labor docente, la cual consiste normalmente en un trabajo con una recompensa implícita.

Un punto a considerar por la institución es el nivel perceptiblemente bajo de la satisfacción en lo que respecta al factor motivacional externo "salario", el cual tuvo una puntuación considerablemente más baja en comparación a los demás factores.

En lo que respecta a la dedicación de los profesores y su relación con los factores motivacionales, como era de esperarse, se encontró que existía una diferencia entre los tipos de dedicación en lo que respecta a la satisfacción general y satisfacción 
con los factores externos. En los análisis específicos de los factores se encontró una diferencia en los factores crecimiento, salario, y condiciones de trabajo. Los profesores a tiempo completo tuvieron puntajes que demostraron una satisfacción más alta que los profesores a tiempo parcial en esos factores. Esto se puede entender a través de la Teoría de la Equidad (Adams, 1965; Greenberg, 1990; Walster et al. 1973). Esta que los individuos comparan las contribuciones que ellos dan a la organización (habilidades, conocimientos, desempeño) y las retribuciones que reciben (salario, oportunidades de crecimiento, etc.) con las contribuciones y retribuciones de otros miembros de la organización. Aquellas personas que perciben que no han sido lo suficientemente retribuidas en comparación a otros trabajadores tratarán de generar equidad a través de cambios en sus actitudes hacia el trabajo, entre ellas, la satisfacción laboral.

En lo que respecta a la categoría de los profesores y su relación con los factores motivacionales, no existió una diferencia en los factores generales. Únicamente se encontró una diferencia con significación estadística en el factor "condiciones de trabajo".

En lo que respecta a la satisfacción en los diferentes factores motivacionales de acuerdo al género de los profesores, llamó la atención encontrar una diferencia en el factor "trabajo en sí". Las docentes mujeres tuvieron puntajes más altos que los docentes varones. Este resultado contrasta los resultados obtenidos en estudios realizados en Estados Unidos (Aguirre 2000; Astin et al. 1997; Hagedorn 2000; Olsen et al. 1995; Perna 2001; Rosser 2005; Smart 1990; Tack y Patitu 1992; Johnsrud y Sadao 1998; Turner y Myers 2000; Trower y Chait 2002; Turner 2002). Las causas de este desacuerdo no son claras, por lo que es necesario realizar más investigaciones para indagar a profundidad sobre este hallazgo.

En lo que respecta al efecto que tienen los factores motivacionales sobre el desempeño docente, los factores supervisión, logro, crecimiento, relaciones y condiciones de trabajo en conjunto, y en ese orden, según este estudio, pueden predecir el desempeño docente en en un $29 \%$.

En lo que respecta a la influencia de los factores específicos sobre el desempeño resulta muy interesante haber encontrado una influencia considerable del factor "supervisión" sobre el desempeño docente. Este resultado llama a la consideración sobre el rol que tienen los decanos, directores de carrera, y coordinadores de carrera y su influencia directa sobre el desempeño de los profesores en el aula de clase.

Finalmente, en lo que respecta a la influencia del salario real sobre el desempeño, no se encontró un resultado del que se pueda inferir su efecto. No obstante, se encontró una moderada influencia del salario real sobre la satisfacción en general. Este hecho también invita a un posterior análisis sobre el efecto indirecto que podría tener el salario sobre el desempeño. 


\section{REFERENCIAS BIBLIOGRÁFICAS}

Adams, J.S. (1965). Inequity in social exchange en Berkowitz, L. Advances in experimental social psychology 2, pp. 267-299. New York: Academic Press.

Aguirre, A. (2000). Women and minority faculty in the academic workplace: Recruitment, retention and academic culture. ASHE-ERIC Higher Education Report 27(6). Washington: American

Association for Higher Education.

Astin, H., Antonio, a., Cress, C., y Astin, A. (1997). Race and ethnicity in the American professoriate, 1995-1996. Los Angeles: Higher Education Research Institute.

Brayfield, Arthur, y Crockett, Walter. (1955). Employee attitudes and Employee Performance. Psychological Bulletin, 52, pp. 396-424

Diener, T. (1985).Job satisfaction and college faculty in two predominantly Black institutions.Journal of Negro Education, 54(4), pp. 558-565.

Greenber, J. (1990). Organizational Justice: Yesterday, Today and Tomorrow. Journal of Management, 16(2), pp. 399-432.

Hackett, R. D., y Guion, R. M. (1985). A re-evaluation of the absenteeism-job satisfaction relationship.Organizational Behavior and Human Decision Processes, 35, pp. 340-381.

Hagedorn, L. (1996). Wage equity and female faculty job satisfaction: The role of wage differentials in a job satisfaction causal model. Research in Higher Education, 37(5), pp. 569-598.

Hagedorn, L. S. (2000). Conceptualizing faculty job satisfaction: components, theories, and outcomes. New Directions for Institutional Research, 27(1), pp. 5-20.

Herzberg, F., Mausner, B. y Snyderman, B. (1959).The Motivation to Work. New York: Wiley.

Herzberg, F. (1968). One More Time: How do you Motivate Employees? Harvard Business Review, pp. 87-96.

Hill, Malcolm. (1986). A theoretical analysis of faculty job satisfaction/dissatisfaction. Educational Research Quarterly, 10(4), pp. 36-44.

Johnsrud, L. K., y Heck, R. H. (1994). A university’s faculty: Predicting those who will stay and those who leave. Journal for Higher Education Management, 10(1), pp. $71-84$.

Johnsrud, L., y Sadao, K. (1998). The common experience of "otherness:" Ethnic and racial minority faculty. Review of Higher Education, 21(4), pp. 315-342.

Judge, Timothy, Thoresen, Carl, Bono, Joyce, Patton, G., (2001). The job satisfactionjob performance relationship: A qualitative and quantitative review. Psychological Bulletin, 127(3), pp. 376-407.

Kanigel, Robert (1997). The One Best Way: Frederick Winslow Taylor and the Enigma of Efficiency. New York: Viking. 
Locke, E. A. (1976). The nature and causes of job satisfaction. Handbook of Industrial and Organizational Psychology (pp. 1297-1349). Chicago: Rand McNally.

Mamiseishvili, K. (2011). Characteristics, job satisfaction, and workplace perceptions of foreign-born faculty at public two-year institutions. Community College Review, 39(1), pp. 26-45.

Olsen, D., Maple, S., y Stage, F. (1995). Women and minority job satisfaction: Professional role interests, professional satisfactions, and institutional fit. Journal of Higher Education, 66(3), pp. 267-293.

Organ, D. y Ryan, K. (1995) A meta-analytic review of attitudinal and dispositional predictors of organizational citizenship behavior. Personnel Psychology. 48, pp. 775-803.

Ostroff, C. (1992). The relationship between satisfaction, attitudes, and performance: An organizational Level Analysis. Journal of Applied Psychology, 77, pp. 963-974.

Perna, L. (2001). Sex differences in faculty salaries: A cohort analysis. Review of Higher Education, 21(4), pp. 315-342.

Rausch, D. K., Ortiz, B. P., Douthitt, R. A., and Reed, L. L. (1989). The academic revolving door: Why do women get caught? CUPA Journal, 40(1), pp. 1-16.

Rosser, V. (2004). Faculty members' intentions to leave: A national study on their worklife and satisfaction. Research in Higher Education, 45(3), pp.223-234.

Rosser, V. (2005). Measuring the change in faculty perceptions over time: An examination of their worklife and satisfaction. Research in Higher Education, 46(1), pp. 81-107.

Serra, Linda (2000). Conceptualizing Faculty Job Satisfaction: Components, Theories, and Outcomes. New Directions for Institutional Research, 105(4), pp. 5-20.

Smart, J. (1990). A causal model of faculty turnover intentions.Research in Higher Education, 31, pp. 405-424.

Smerek, R. y Peterson, M. (2007). Examining Herzberg's Theory: Improving Job Satisfaction among Non-academic Employees at a University.Research in Higher Education, 48(2), pp. 229-250.

Tack, M., y Patitu, C. (1992). Faculty Job Satisfaction: Women and Minorities in Peril, ERIC Clearinghouse on Higher Education, Washington, DC.

Trower, C., y Chait, R. (2002). Faculty diversity: Too little for too long. Harvard Magazine, 98, pp. 33-37.

Turner, C. (2002). Women of color in academe: Living with multiple marginality. Journal of Higher Education, 73(1), pp. 74-93.

Turner, C., y Myers, S. (2000).Faculty of color in academe: Bittersweet success. Needham Heights: Allyn\& Bacon.

Walster, E., Berscheid, E., y Walster, G. W. (1973). New directions in equity research Journal of Personality and Social Psychology, 25, pp. 151-176. 
